\title{
p27-V109G Polymorphism Is Not Associated with the Risk of Prostate Cancer: A Case-Control Study of Han Chinese Men in Central China
}

\author{
Meng Zhang $\mathbb{D}^{1,2,3}$ Qianjun Liang, ${ }^{1,2,3}$ Ligang Zhang, ${ }^{1,2,3}$ Zongyao Hao, ${ }^{1,2}$ Jun Zhou, ${ }^{1,2}$ \\ Li Zhang $\oplus^{1,2}$ Song Fan, ${ }^{1,2}$ and Chaozhao Liang $\oplus^{1,2}$ \\ ${ }^{1}$ Department of Urology, The First Affiliated Hospital of Anhui Medical University, Hefei, China \\ ${ }^{2}$ Institute of Urology, Anhui Medical University, Hefei, China \\ ${ }^{3}$ Graduate School, Anhui Medical University, Hefei, China
}

Correspondence should be addressed to Chaozhao Liang; liang_chaozhao@ahmu.edu.cn

Received 22 August 2017; Accepted 28 January 2018; Published 20 March 2018

Academic Editor: Marco E. M. Peluso

Copyright (c) 2018 Meng Zhang et al. This is an open access article distributed under the Creative Commons Attribution License, which permits unrestricted use, distribution, and reproduction in any medium, provided the original work is properly cited.

\begin{abstract}
Objective. We conducted an update meta-analysis aiming to verify the association between $p 27-\mathrm{V} 109 \mathrm{G}$ polymorphism and cancer risk, particular for prostate cancer (PCa). Then, we conducted a case-control study of Han Chinese in central China to verify the evidence-based results. Methods. Relevant studies were collected from diverse databases up to March 2017. In addition, a hospital-based (H-B) case-control study enrolling $90 \mathrm{PCa}$ patients and 140 healthy controls was included to verify these evidence-based findings. Genetic risk was calculated by odds ratio (OR) with its corresponding 95\% confidence interval (CI). The p27-V109G polymorphism was determined by MassARRAY genotyping method. Results. Finally, twenty-four published studies comprising 9627 cases and 12,102 controls were enrolled for the current meta-analysis. Overall analysis suggested that p27-V109G polymorphism decreased overall cancer risk in allelic contrast, heterozygote, and dominant models. When stratified analysis was conducted by ethnicity, data revealed that $p 27-\mathrm{V} 109 \mathrm{G}$ polymorphism was associated with a decreased cancer risk in Caucasians. Highlighted in the subgroup analysis by cancer type, we uncovered a significantly decreased risk of PCa in allelic contrast, dominant, homogeneous, and recessive models. However, in the validation case-control study, we failed to uncover a positive association between p27-V109G polymorphism and PCa risk. In addition, negative results were also identified when subgroup analyses were stratified by age, tumor grade, tumor stage, PSA levels, and other measurements. Conclusion. Although evidence-based results suggest that $227-\mathrm{V} 109 \mathrm{G}$ polymorphism plays a protective role in overall cancer risk, particularly for PCa, our case-control study failed to validate any association between this particular polymorphism and PCa risk.
\end{abstract}

\section{Introduction}

Prostate cancer ( $\mathrm{PCa}$ ) is the most common malignancy in the urogenital system of men and has a high lethality. In the Asian population, although $\mathrm{PCa}$ incidence remains at a relatively low level, it keeps increasing $[1,2]$. Therefore, it is important to improve the diagnosis and treatment level of PCa. At present, like other cancers, pathogenesis of PCa is not clear [3], and lifestyle behaviors [4-6] and genetic factors [7-10] are considered as risk factors. In addition, study has shown that the incidence of PCa is about $57 \%$ gene-related [11], and of them, $p 27$ is one of the extensively studied genes.
Abnormal cell cycle regulation can lead to the occurrence of cancer [12]. As an important cell cycle regulatory gene, the p27 (also known as CDKN1B) controls the cell cycle check from G1 to S transition [13]. Downregulation of the p27 expression is associated with various malignant neoplasms, including PCa $[14,15]$. According to the dbSNP database, there are at least 59 mutated sites in the p27. Previous researches have concentrated on one of the important polymorphisms, namely p27-V109G (rs2066827), formed in a polymorphism at codon $109(\mathrm{~T}>\mathrm{G})$ which caused substitution of glycine from valine and potential functional protein alteration [16]. The protein alteration, caused by 
p27-V109G polymorphism, leads to the reduction of the p27 expression, weakens negative regulation of the cell cycle, and induces tumorigenesis $[15,17]$.

Recently, some publications have reported the association between p27-V109G polymorphism and cancer risk, whereas conclusion was inconsistent, particularly for PCa. For example, some of them suggested that the p27-V109G polymorphism was associated with a decreased risk of cancer $[18,19]$, including PCa, while one research indicated that the p27-V109G polymorphism was not associated with PCa [20]. Therefore, in order to find out the precise relationship between this polymorphism and cancer risk (particularly for PCa), we performed an updated meta-analysis. In addition, we also conducted a hospital-based (H-B) case-control studies to further validate these evidence-based findings.

\section{Materials and Methods}

\subsection{Updated Meta-Analysis}

2.1.1. Search Strategy and Study Selection. Relevant literatures were collected from PubMed, Google Scholar, Web of Science, CNKI, and Wanfang Data databases up to March 2017, using the following retrieval types: ("p27" OR “CDKN1B” OR “V109G” OR “rs2066827”) AND (“polymorphism" OR "mutation" OR "variant” OR "SNP” OR "genotype") AND (“cancer" OR “carcinoma” OR "tumor”). Articles satisfied with the following inclusion criteria would be enrolled: (1) studies investigated the association between p27-V109G polymorphism and cancer risk, (2) designed as case-control, (3) genotype and allele frequencies can be extracted to calculate odds ratio (OR) and 95\% confidence interval (CI), and (4) full-text studies published in English or Chinese. The major exclusion criteria were (1) case reports, case-only, or Review studies and (2) studies without raw data for $p 27-\mathrm{V} 109 \mathrm{G}$ polymorphism (or contacting the corresponding author still did not obtain the necessary original data). In addition, discrepancies will be resolved by consensus.

2.1.2. Statistical Analysis. Statistical analyses were conducted by STATA 12.0 software (StataCorp., College Station, TX). The common measure of association across studies, ORs corresponding with $95 \%$ CIs were applied to evaluate cancer susceptibility. Pooled ORs of four genetic models were computed: allelic contrast, heterozygote, homozygote dominant, and recessive models. Chi-square test-based $\mathrm{Q}$ test was adopted to measure the heterogeneity across studies. In addition, the Hardy-Weinberg equilibrium (HWE) of control groups was calculated using chi-square test. The stability of results was estimated by sensibility analysis, and publication bias was estimated by Begg's funnel plot and Egger's regression test. Statistically significance was set at $P<0.05$.

\subsection{Case-Control Study}

2.2.1. Study Subjects. In this study, both patients and controls were recruited from the Department of Urology, the First Affiliated Hospital of Anhui Medical University. A total of $90 \mathrm{PCa}$ patients and 140 controls were enrolled for this work between September 2014 and July 2016. Patients were newly diagnosed and pathologically confirmed as PCa, and control groups were almost age-matched, with normal prostatespecific antigen (PSA) levels $(<4 \mathrm{ng} / \mathrm{ml})$, without cancer history. Each participant signed an informed consent. We also collected relevant clinicopathologic data, as well as body mass index (BMI), systolic blood pressure (SBP), and serum PSA levels from medical record review.

2.2.2. Genotyping Analysis. The genomic DNA of each participant was extracted from blood sample by using commercially DNA extraction kits (Qiagen, catalog: 51106). For each DNA sample, p27-V109G polymorphism was detected by MassARRAY (Sequenom, San Diego, CA). Primer was designed by Assay Design software package (Sequenom) (5' -TGCAGACCCGGAGAAAG-3', $5^{\prime}$-CCGC TAACCCCGTCTGG- ${ }^{\prime}$ ). Details of the experiment process were referring to Qin et al.'s [21] work. And results were analyzed by TYPER 4.0 software (Sequenom).

2.2.3. Statistical Analyses. HWE of controls was calculated by chi-square statistics. The cancer risk, which was assessed by ORs with 95\% CI, was calculated by using logistic regression models; otherwise, calculation results were adjusted for age and BMI. $P<0.05$ was regarded as statistically significant. Differences of different clinical stages, Gleason grades, and PSA levels in PCa patients were also calculated by using the same statistic methods mentioned above. Moreover, in order to control possible confounding, logistic regression model was conducted to adjust these demographic characteristics. All statistical analyses were executed based on SPSS 17.0 version (SPSS Inc., Chicago, United States).

\section{Results}

3.1. Meta-Analysis. We performed an updated metaanalysis to evaluate the association between p27-V109G polymorphism and cancer risk. After restricting the selection process referring to the inclusion and exclusion criteria, a total of 24 articles were enrolled (Supplementary Table 1) $[18,20,22-41]$. In addition, four studies were not consistent with HWE [18, 30, 38, 40]. Publication selection process is presented in Supplementary Figure 1.

Overall, a significant decreased risk of overall cancer was identified in three genetic models ( $\mathrm{G}$ versus $\mathrm{T}$ : $\mathrm{OR}=0.859$, 95\% CI: $0.764-0.967, P=0.012$; GT versus TT: $\mathrm{OR}=0.881$, 95\% CI: $0.783-0.991, P=0.035$; and GT/GG versus TT: $\mathrm{OR}=0.829,95 \%$ CI: 0.729-0.942, $P=0.004$ ) (Supplementary Table 2). In the hierarchical analysis by ethnicity, data revealed that $p 27-\mathrm{V} 109 \mathrm{G}$ polymorphism was associated with a decreased risk of overall cancer in Caucasians instead of Asians (GT/GG versus TT: OR $=0.913$, 95\% CI: $0.847-$ $0.983, P=0.016)$. Highlighted in the subgroup analysis by cancer type, we also uncovered a significant decreased risk of PCa in four genetic models ( $G$ versus $\mathrm{T}$ : $\mathrm{OR}=0.537$, 95\% CI: $0.365-0.789, P=0.002$; GT/GG versus TT: OR= 0.554, 95\% CI: $0.351-0.876, P=0.012$; GG versus TT: $\mathrm{OR}=$ 0.320, 95\% CI: $0.129-0.791, P=0.014$; and GG versus GT/ TT: $\mathrm{OR}=0.363,95 \% \mathrm{CI}: 0.149-0.886, P=0.026)$. 
TABLE 1: Characteristics of PCa cases and controls in a Chinese population.

\begin{tabular}{|c|c|c|c|c|}
\hline Characteristic & Case & Control & $P$ value $^{\mathrm{a}}$ & OR (95\% CI) \\
\hline Sample size & $N=90$ & $N=140$ & & \\
\hline Age (years $\pm S D)$ & $73.00 \pm 7.64$ & $67.66 \pm 6.69$ & $<0.001$ & \\
\hline Age (years) & & & $<0.001$ & $3.715(2.128-6.487)$ \\
\hline$\leq 70$ & 34 & 97 & & \\
\hline$>70$ & 56 & 43 & & \\
\hline BMI & & & 0.098 & $1.661(0.910-3.030)$ \\
\hline$\leq 23$ & 21 & 47 & & \\
\hline$>23$ & 69 & 93 & & \\
\hline Systolic blood pressure & & & 0.787 & $1.076(0.633-1.827)$ \\
\hline$<140$ & 44 & 71 & & \\
\hline$\geq 140$ & 46 & 69 & & \\
\hline
\end{tabular}

${ }^{a}$ Two-sided $\chi^{2}$ test for the distributions between the cases and controls.

TABLE 2: Relationship between the p27-V109G polymorphism and PCa risk.

\begin{tabular}{|c|c|c|c|c|c|}
\hline Genotype & Cases & Controls & $P$ value $^{\mathrm{a}}$ & OR (95\% CI) & OR $(95 \% \mathrm{CI})^{\mathrm{b}}$ \\
\hline TT & 77 & 127 & & 1.00 (reference) & \\
\hline TG & 13 & 13 & 0.231 & $1.649(0.727-3.742)$ & $2.132(0.881-5.155)$ \\
\hline GG & 0 & 0 & - & - & - \\
\hline TG/GG & 13 & 13 & 0.231 & $1.649(0.727-3.742)$ & $2.132(0.881-5.155)$ \\
\hline \multicolumn{6}{|l|}{ Allele } \\
\hline $\mathrm{T}$ & 167 & 267 & 0.246 & 1.00 (reference) & \\
\hline G & 13 & 13 & & $1.599(0.724-3.532)$ & - \\
\hline
\end{tabular}

${ }^{\mathrm{a}}$ Two-sided $\mathrm{x} 2$ test for the distributions between the cases and controls; ${ }^{\mathrm{b}}$ adjusting for age and body mass index (BMI).

In addition, sensitivity analysis suggested that overall results did not change obviously and remained robust after removing any of the individual research, which proved the stability of our results (Supplementary Table 3). Begg's funnel plot and Egger's regression test were executed to assess publication bias, and final results suggested no publication bias existed (Supplementary Figure 2).

Furthermore, in order to validate the evidence-based findings obtained above, which suggested that p27-V109G polymorphism was a protective factor for $\mathrm{PCa}$, we further conducted a H-B case-control study of Han Chinese men in central China. Details of the case-control work were demonstrated as follows.

3.2. Characteristics of the Study Population in the CaseControl Study. Table 1 summarized the detailed demographic characteristics of recruited cases and controls. Mean ages of patients and controls were $73.00 \pm 7.64$ years (range from 48 to 87 years) and $67.66 \pm 6.69$ years (range from 60 to 95 years), respectively. The ages of the two groups showed a statistically significant difference $(P<0.001)$. No significant difference was uncovered in the BMI and blood pressure subgroups.

3.3. p27-V109G Polymorphism with Risk of PCa. The genotypic distributions of $p 27-\mathrm{V} 109 \mathrm{G}$ polymorphisms were shown in Table 2. The genotype frequency of controls was conformed to HWE $(P=0.565)$. Compared to TT genotypes, the variant TG and GG genotypes were not associated with PCa risk after adjusting for other potential covariates. The frequency of p27-V109G genotypes was similar between PCa cases and controls (Table 2). In addition, a negative result was also uncovered when stratified analyses were conducted by different age groups and BMI (Table 3 ).

For genotypic comparison, patients with different Gleason grades (based on degree of differentiation between cells, low grade $<7$ and high grade $>7$ ), clinical stages [based on international tumor-node-metastasis (TNM) system for PCa, localized and advanced], and PSA levels (PSA $\leq 20 \mathrm{ng} / \mathrm{ml}$ and PSA $>20 \mathrm{ng} / \mathrm{ml}$ ) were subcategorized into 2 subgroups. However, no significant association was also observed in all these subgroups (Table 4 ).

\section{Discussion}

As we all know, etiology of PCa remains unclear. It has been postulated that many factors can result in malignant neoplasms. In recent decades, many researchers drew attention to the association between genetic polymorphisms and cancer susceptibility $[42,43]$. Since then, SNP has been found contributed to the incidence of cancer [44], including PCa.

According to the literatures, several variants located in p27 play an inherited role in cancer. p27 mainly include two polymorphisms [45], called rs2066827 (109T/G) and 
TABLE 3: Stratification analysis of p27-V109G genotypes and risk of PCa.

\begin{tabular}{|c|c|c|c|c|c|}
\hline Group & $N$ (case/control) & TT & TG/GG & $P$ value $^{\mathrm{a}}$ & OR (95\% CI) \\
\hline \multicolumn{6}{|l|}{ Age } \\
\hline$\leq 70$ & $34 / 97$ & $28 / 85$ & $6 / 12$ & 0.444 & $1.518(0.521-4.421)$ \\
\hline$>70$ & $56 / 43$ & $49 / 42$ & $7 / 1$ & 0.100 & $6.000(0.709-50.765)$ \\
\hline \multicolumn{6}{|l|}{ BMI } \\
\hline$\leq 23$ & $21 / 47$ & $19 / 42$ & $2 / 5$ & 0.889 & $0.884(0.157-4.973)$ \\
\hline$>23$ & $69 / 93$ & $58 / 85$ & $11 / 8$ & 0.157 & $2.015(0.764-5.315)$ \\
\hline \multicolumn{6}{|c|}{ Systolic blood pressure } \\
\hline$<140$ & $44 / 71$ & $37 / 62$ & $7 / 9$ & 0.627 & $1.303(0.448-3.793)$ \\
\hline$\geq 140$ & $46 / 69$ & $40 / 65$ & $6 / 4$ & 0.188 & $2.437(0.648-9.171)$ \\
\hline
\end{tabular}

${ }^{a}$ Two-sided $\chi^{2}$ test for the distributions between TT and TT/GG.

TABLE 4: p27-V109G polymorphism and clinic pathological characteristics in Chinese patients with PCa.

\begin{tabular}{|c|c|c|c|c|c|}
\hline Group & TT (\%) & TG/GG (\%) & $P$ value $^{\mathrm{a}}$ & OR (95\% CI) & OR $(95 \% \mathrm{CI})^{\mathrm{b}}$ \\
\hline Clinical $^{\mathrm{c}}$ & & & 0.161 & $0.222(0.027-1.816)$ & - \\
\hline Localized & 56 & 12 & & & \\
\hline Advanced & 21 & 1 & & & \\
\hline Gleason & & & 0440 & $1.600(0.453-5.652)$ & $1.651(0.463-5.890)$ \\
\hline$>7$ & 32 & 4 & & & \\
\hline$\leq 7$ & 45 & 9 & & & \\
\hline Total PSA (ng/ml) & & & 0.972 & $0.875(0.269-2.848)$ & $0.978(0.290-3.296)$ \\
\hline$>20$ & 33 & 6 & & & \\
\hline$\leq 20$ & 44 & 7 & & & \\
\hline
\end{tabular}

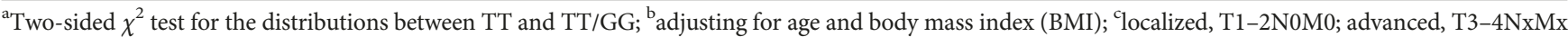
or TxN1Mx or TxNxM1. Clinical staging according to the international TNM system for prostate cancer.

rs34330 (-79 C/T). Some researchers have shown that p27 polymorphism has a protective effect on cancers $[18,32,33]$, while other studies proved that it did not correlate with the risk of cancer in the general population [24, 46, 47]. For this reason, Lu et al. [48] conducted a meta-analysis including 17 case-control studies enrolling 9038 cases and 11,596 controls and observed that the 109T/G TG genotype had a protective role on cancer. And Cheng et al. [49] also conducted a meta-analysis aiming to verify the association between $p 27$ rs34330 polymorphism and cancer risk; they suggested that rs34330 polymorphism was related to an increased risk of cancer. Here, we reconducted an updated meta-analysis containing 24 case-control studies comprising of 9627 cases and 12,102 controls and identified that $p 27-$ V109G polymorphism was associated with a decreased risk of cancer, which was consistent with $\mathrm{Lu}$ et al.'s work [48]. In addition, when stratification analysis was conducted by ethnicity, we uncovered a negative result between $p 27$ V109G polymorphism and cancer risk in Asians, but it seemed to work as a protective effect in Caucasians. Moreover, in stratification analysis by cancer type, we also uncovered a significant decreased risk of PCa.

Previously, several publications have studied the association between rs2066827 polymorphism and PCa risk in different ethnicities [32, 46, 50, 51]. In 2003, Kibel et al. [19] designed a study enrolling 96 cases and 105 controls of Caucasian origin, demonstrating that this polymorphism was associated with a decreased risk of PCa. In 2015, Han et al. [18] conducted a study based on 70 cases and 70 controls in an Asian population and also found that $p 27$ V109G polymorphism played a protective role in PCa risk. However, results from our case-control study were not consistent with the studies mentioned above, as well as the evidence-based results derived from meta-analysis, but consistent with Huang et al.'s [20] work, which contained 190 patients and 292 controls of Asian descent, suggesting that $p 27-V 109 \mathrm{G}$ polymorphism was not associated with $\mathrm{PCa}$. In addition, our results also suggested that no significant associations were also uncovered between this polymorphism and clinicopathologic features of PCa. Hereditary heterogeneity of subjects, different polymorphisms of $p 27$, or inadequate adjustment for confounding factors can lead to the above inconsistency. Apart from these, sample size may also affect the results.

Although we have carried out a comprehensive retrieval on diverse databases, and obtained a convincible evidencebased results, which were also validated by a H-B based case-control study, there are still several limitations that should be noted in our study. First, only studies published in English or Chinese were included, which will result in potential study selection bias. Second, for PCa, they are only three case-control studies included and, of them, two generated from an Asian (Chinese) population. Although we obtained a positive association between $p 27$ - 
V109G polymorphism and PCa risk, the enrolled sample size was relatively small, which may contribute to false-positive or false-negative findings. Thirdly, we only took the $p 27$ genetic variant into account; other genetic factors, such as gene-gene and gene-environment interactions, can also influence cancer risk. Fourthly, for subgroup analysis, lack of adequate eligible studies may lead to insufficient statistical power, limiting the ability to identify weakly positive results. Fifthly, for the case-control study, we only made a genetic analysis of the p27-V109G in a Chinese population and the sample size was also limited. Moreover, the age of the cases and controls and the existing statistical significance may affect the results of the current work.

Taken together, the current work suggests that p27V109G polymorphism may not be the risk factor for PCa, a result inconsistent with our meta-analysis work. Although meta-analysis is a powerful tool to cumulate and summarize the knowledge in a research field through statistical instruments, it can also be limited by the number of studies and sample sizes enrolled. Nevertheless, all these findings may require larger sample-size studies in the Chinese Han population to further validate them.

\section{Conflicts of Interest}

There is no conflict of interest in this article.

\section{Authors' Contributions}

All authors agreed to publish this work and contributed to the design, data extraction, data analysis, chart drawing drafting, and revising of the article. All authors are committed to being responsible for their work. Meng Zhang, Qianjun Liang, and Ligang Zhang performed the experiments, literature search, data extraction, and statistical analysis and wrote the manuscript. Zongyao Hao, Jun Zhou, Ligang Zhang, Song Fan, and Chaozhao Liang supervised the literature search, data extraction, and analysis. Meng Zhang, Chaozhao Liang, and Qianjun Liang reviewed the manuscript. Meng Zhang and Qianjun Liang contributed equally to the work.

\section{Supplementary Materials}

Supplementary Table 1: main characteristics of all studies included in the meta-analysis. Supplementary Table 2: the overall analyses of $p 27-\mathrm{V} 109 \mathrm{G}$ polymorphism and cancer risk. Supplementary Table 3: details of the sensitivity analyses for the p27-V109G polymorphism and cancer risk. Supplementary Figure 1: flow chart shows study selection process. Supplementary Figure 2: funnel plot of publication bias analysis for the associations between the p27-V109G polymorphism and cancer risk (GT+GG versus TT). (Supplementary Materials)

\section{References}

[1] Y. Amiya, Y. Yamada, M. Sugiura et al., "Outcomes of patients older than 75 years with non-metastatic prostate cancer," Asian Journal of Urology, vol. 4, no. 2, pp. 102-106, 2017.
[2] K. Zhang, C. H. Bangma, and M. J. Roobol, "Prostate cancer screening in Europe and Asia," Asian Journal of Urology, vol. 4, no. 2, pp. 86-95, 2017.

[3] J.-L. Bai, M.-H. Zheng, X. Xia, M. ter-Minassian, Y. P. Chen, and F. Chen, "MTHFR C677T polymorphism contributes to prostate cancer risk among Caucasians: a meta-analysis of 3511 cases and 2762 controls," European Journal of Cancer, vol. 45, no. 8, pp. 1443-1449, 2009.

[4] X.-T. Zeng, W.-D. Leng, C. Zhang, J. Liu, S.-Y. Cao, and W. Huang, "Meta-analysis on the association between toothbrushing and head and neck cancer," Oral Oncology, vol. 51, no. 5, pp. 446-451, 2015.

[5] K. E. N. Petersen, N. F. Johnsen, A. Olsen et al., "The combined impact of adherence to five lifestyle factors on all-cause, cancer and cardiovascular mortality: a prospective cohort study among Danish men and women," British Journal of Nutrition, vol. 113, no. 5, pp. 849-858, 2015.

[6] P. L. Gawade, K. C. Oeffinger, C. A. Sklar et al., "Lifestyle, distress, and pregnancy outcomes in the childhood cancer survivor study cohort," American Journal of Obstetrics \& Gynecology, vol. 212, no. 1, pp. 47.e1-47.e10, 2015.

[7] C. Zhang, W. Wang, H. Zhang, L. Wei, and S. Guo, "Association of FCGR2A rs1801274 polymorphism with susceptibility to autoimmune diseases: a meta-analysis," Oncotarget, vol. 7, no. 26, pp. 39436-39443, 2016.

[8] M. J. Wang, Y. J. Luo, Z. Y. Shi et al., “The associations between MDM4 gene polymorphisms and cancer risk," Oncotarget, vol. 7, no. 34, pp. 55611-55623, 2016.

[9] H.-L. Wang, X. Lu, X. Yang, and N. Xu, "Association of MBL2 exon1 polymorphisms with high-risk human papillomavirus infection and cervical cancers: a meta-analysis," Archives of Gynecology and Obstetrics, vol. 294, no. 6, pp. 1109-1116, 2016.

[10] F. Liu, K. Dear, L. Huang et al., "Association between microRNA-27a rs895819 polymorphism and risk of colorectal cancer: a meta-analysis," Cancer Genetics, vol. 209, no. 9, pp. 388-394, 2016.

[11] M. R. Safarinejad, N. Shafiei, and S. Safarinejad, "Relationship of insulin-like growth factor (IGF) binding protein-3 (IGFBP3) gene polymorphism with the susceptibility to development of prostate cancer and influence on serum levels of IGF-I, and IGFBP-3," Growth Hormone \& IGF Research, vol. 21, no. 3, pp. 146-154, 2011.

[12] D. Hanahan and R. A. Weinberg, "Hallmarks of cancer: the next generation," Cell, vol. 144, no. 5, pp. 646-674, 2011.

[13] A. Alkarain and J. Slingerland, "Deregulation of p27 by oncogenic signaling and its prognostic significance in breast cancer," Breast Cancer Research, vol. 6, no. 1, pp. 13-21, 2003.

[14] S. Molatore, E. Kiermaier, C. B. Jung et al., "Characterization of a naturally-occurring $\mathrm{p} 27$ mutation predisposing to multiple endocrine tumors," Molecular Cancer, vol. 9, no. 1, p. 116, 2010.

[15] K. Tomoda, Y. Kubota, and J. Kato, "Degradation of the cyclindependent-kinase inhibitor p27 $7^{\mathrm{Kip} 1}$ is instigated by Jab1," Nature, vol. 398, no. 6723, pp. 160-165, 1999.

[16] H. Cavé, E. Martin, I. Devaux, and B. Grandchamp, "Identification of a polymorphism in the coding region of the $\mathrm{p} 27^{\mathrm{Kip} 1}$ gene," Annales De Genetique, vol. 38, no. 2, p. 108, 1995.

[17] S.-L. Guo, Z. Peng, X. Yang et al., "miR-148a promoted cell proliferation by targeting p27 in gastric cancer cells," International Journal of Biological Sciences, vol. 7, no. 5, pp. 567-574, 2011. 
[18] Q. H. Han, Z. J. Shan, J. T. Hu, N. Zhang, and X. P. Zhang, "Relationship between gene polymorphisms and prostate cancer risk," Asian Pacific Journal of Tropical Medicine, vol. 8, no. 7, pp. 569-573, 2015.

[19] F. Wei, J. Xu, L. Tang et al., "p27 $7^{\mathrm{Kip} 1}$ V109G polymorphism and cancer risk: a systematic review and meta-analysis," Cancer Biotherapy \& Radiopharmaceuticals, vol. 27, no. 10, pp. 665-671, 2012.

[20] S. P. Huang, C. C. Yu, C. C. Liu, T. T. Wu, C. H. Huang, and M. T. Wu, "CDKN1B V109G polymorphism frequency and prostate cancer risk in Taiwan," Urologia Internationalis, vol. 81, no. 1, pp. 36-40, 2008.

[21] Y. R. Qin, L. Fu, P. C. Sham et al., "Single-nucleotide polymorphism-mass array reveals commonly deleted regions at 3p22 and 3p14.2 associate with poor clinical outcome in esophageal squamous cell carcinoma," International Journal of Cancer, vol. 123, no. 4, pp. 826-830, 2008.

[22] A. S. Kibel, B. K. Suarez, J. Belani et al., "CDKN1A and CDKN1B polymorphisms and risk of advanced prostate carcinoma," Cancer Research, vol. 63, no. 9, pp. 2033-2036, 2003.

[23] S. A. Gayther, H. Song, S. J. Ramus et al., "Tagging single nucleotide polymorphisms in cell cycle control genes and susceptibility to invasive epithelial ovarian cancer," Cancer Research, vol. 67, no. 7, pp. 3027-3035, 2007.

[24] J. C. Figueiredo, J. A. Knight, S. Cho et al., "Polymorphisms cMyc-N11S and p27-V109G and breast cancer risk and prognosis," BMC Cancer, vol. 7, no. 1, p. 99, 2007.

[25] W. Guo, Y. J. Cui, S. M. Fang, Y. Li, N. Wang, and J. H. Zhang, "Association of polymorphisms of p21cip1 and p27kip1 genes with susceptibilities of esophageal squamous cell carcinoma and gastric cardiac adenocarcinoma," Chinese Journal of Cancer, vol. 25, no. 2, pp. 194-199, 2006.

[26] Y. U. Jiang, S. Feng, J. Huang et al., "Correlation between polymorphisms of p21 and p27 gene and gynecological tumor," Shandong Medical Journal, vol. 48, no. 33, pp. 4-5, 2008.

[27] X. Jin, S. Kang, N. Wang, Y. P. Xing, and Y. Li, "Single nucleotide polymorphisms in cell cycle regulator p21 and p27 genes are associated with susceptibility to epithelial ovarian cancer," Zhonghua Fu Chan Ke Za Zhi, vol. 43, no. 3, pp. 209-212, 2008.

[28] G. Li, E. M. Sturgis, L.-E. Wang et al., "Association between the V109G polymorphism of the p27 gene and the risk and progression of oral squamous cell carcinoma," Clinical Cancer Research, vol. 10, no. 12, pp. 3996-4002, 2004.

[29] Z. Li, Y. Ma, B. Li, Y Zhang, Y Zhang, and Y Li, "Study of correlation between polymorphisms of p21 and p27 genes and non-small cell lung cancer," Chinese Journal of Cancer Prevention Treatment, vol. 14, no. 14, pp. 1046-1049, 2007.

[30] F. Liu, Y. G. Wei, L. M. Luo et al., "Genetic variants of p21 and p27 and hepatocellular cancer risk in a Chinese Han population: a case-control study," International Journal of Cancer Journal, vol. 132, no. 9, pp. 2056-2064, 2013.

[31] F. Z. Mohamed, Y. M. Hussien, M. M. Albakry, R. H. Mohamed, and N. Mohamed Said, "Role of DNA repair and cell cycle control genes in ovarian cancer susceptibility," Molecular Biology Reports, vol. 40, no. 5, pp. 3757-3768, 2013.

[32] D. Pasquali, L. Circelli, A. Faggiano et al., "CDKN1B V109G polymorphism a new prognostic factor in sporadic medullary thyroid carcinoma," European Journal of Endocrinology, vol. 164, no. 3, pp. 397-404, 2011.
[33] T. Sekiya, M. D. Bronstein, K. Benfini et al., "P27 variant and corticotropinoma susceptibility: a genetic and in vitro study," Endocrine-Related Cancer, vol. 21, no. 3, pp. 395404, 2014.

[34] M. A. Yu-Quan, X. G. Yang, H. Liu, and B. Q. Li, "Influence of p21 and p27 gene polymorphisms on development and lymphatic metastasis of esophageal squamous cell carcinoma," Journal of the Fourth Military Medical University, vol. 29, no. 17, pp. 1600-1603, 2008.

[35] Z. S. Li, B. Q. Li, and G. C. Wang, "Study on the correlation between polymorphisms of p21 and p27 genes and the genetic susceptibility of non-small cell lung carcinoma in women," Hebei Medical Journal, vol. 32, no. 3, pp. 272-275, 2010.

[36] Y. Zhang, Z. Q. Luo, X. J. Du, and Y. H. Li, "The association of p16, p21, p27 polymorphism with susceptibility to ovarian cancer," National Medical Frontiers of China, vol. 8, no. 9, pp. 2728, 2013.

[37] K. E. Driver, H. Song, F. Lesueur et al., “Association of singlenucleotide polymorphisms in the cell cycle genes with breast cancer in the British population," Carcinogenesis, vol. 29, no. 2, pp. 333-341, 2008.

[38] A. A. Ferrando, M. Balbín, A. M. Pendás, F. Vizoso, G. Velasco, and C. López-Otín, "Mutational analysis of the human cyclindependent kinase inhibitor p $27^{\text {kip } 1}$ in primary breast carcinomas," Human Genetics, vol. 97, no. 1, pp. 91-94, 1996.

[39] H. Ma, G. Jin, Z. Hu et al., "Variant genotypes of CDKN1A and $C D K N 1 B$ are associated with an increased risk of breast cancer in Chinese women," International Journal of Cancer, vol. 119, no. 9, pp. 2173-2178, 2006.

[40] R. Naidu, Y. C. Har, and N. A. Taib, "P27 V109G polymorphism is associated with lymph node metastases but not with increased risk of breast cancer," Journal of Experimental \& Clinical Cancer Research, vol. 26, no. 1, pp. 133-140, 2007.

[41] V. Ü. Onay, L. Briollais, J. A. Knight et al., "SNP-SNP interactions in breast cancer susceptibility," BMC Cancer, vol. 6 , no. 1, p. 114, 2006.

[42] A. Hossain, G. M. M. Murshid, M. N. H. Zilani et al., "TP53 codon 72 polymorphism and breast cancer risk in Bangladeshi population," Breast Cancer, vol. 24, no. 4, pp. 571-578, 2017.

[43] N. Putthanachote, S. Promthet, K. Suwanrungruan et al., "XRCC1 gene polymorphism, clinicopathological characteristics and stomach cancer survival in Thailand," Asian Pacific Journal of Cancer Prevention, vol. 16, no. 14, pp. 6111-6116, 2015.

[44] R. V. Lloyd, L. A. Erickson, L. Jin et al., "p27 $7^{\text {kip1 }}$ : a multifunctional cyclin-dependent kinase inhibitor with prognostic significance in human cancers," The American Journal of Pathology, vol. 154, no. 2, pp. 313-323, 1999.

[45] G. P. Yu, Q. Y. Xiao, Z. Q. Shi et al., "Genetic polymorphisms in apoptosis-related genes and the prognosis of hepatocellular carcinoma," American Journal of Cancer Research, vol. 5, no. 10, pp. 3249-3259, 2015.

[46] J. Chen, C. K. Amos, K. W. Merriman et al., "Genetic variants of $p 21$ and $p 27$ and pancreatic cancer risk in non-Hispanic whites: a case-control study," Pancreas, vol. 39, no. 1, pp. $1-$ 4, 2010.

[47] T. Schöndorf, L. Eisele, U. J. Göhring et al., “The V109G polymorphism of the $\mathrm{p} 27$ gene $C D K N 1 B$ indicates a worse outcome in node-negative breast cancer patients," Tumor Biology, vol. 25, no. 5-6, pp. 306-312, 2004. 
[48] Y. Lu, K. Gao, M. Zhang et al., "Genetic association between CDKN1B rs2066827 polymorphism and susceptibility to cancer," Medicine, vol. 94, no. 46, article e1217, 2015.

[49] X. K. Cheng, X. J. Wang, X. D. Li, and X. Q. Ren, "Genetic association between the cyclin-dependent kinase inhibitor gene p27/Kip1 polymorphism (rs34330) and cancer susceptibility: a meta-analysis," Scientific Reports, vol. 7, article 44871, 2017.

[50] A. Murali, K. R. Nalinakumari, S. Thomas, and S. Kannan, "Association of single nucleotide polymorphisms in cell cycle regulatory genes with oral cancer susceptibility," British Journal of Oral and Maxillofacial Surgery, vol. 52, no. 7, pp. 652-658, 2014.

[51] G. Lima, E. Santos, H. Angelo et al., “Association between p21 Ser31Arg polymorphism and the development of cervical lesion in women infected with high risk HPV," Tumor Biology, vol. 37, no. 8, pp. 10935-10941, 2016. 


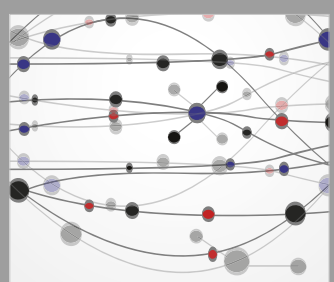

The Scientific World Journal
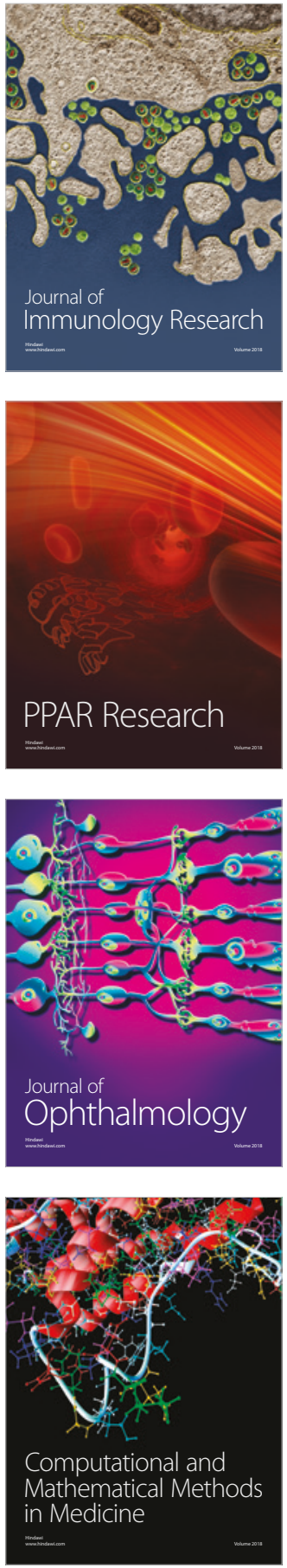

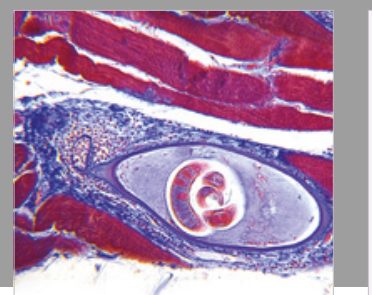

Gastroenterology Research and Practice

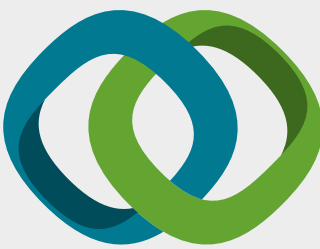

\section{Hindawi}

Submit your manuscripts at

www.hindawi.com
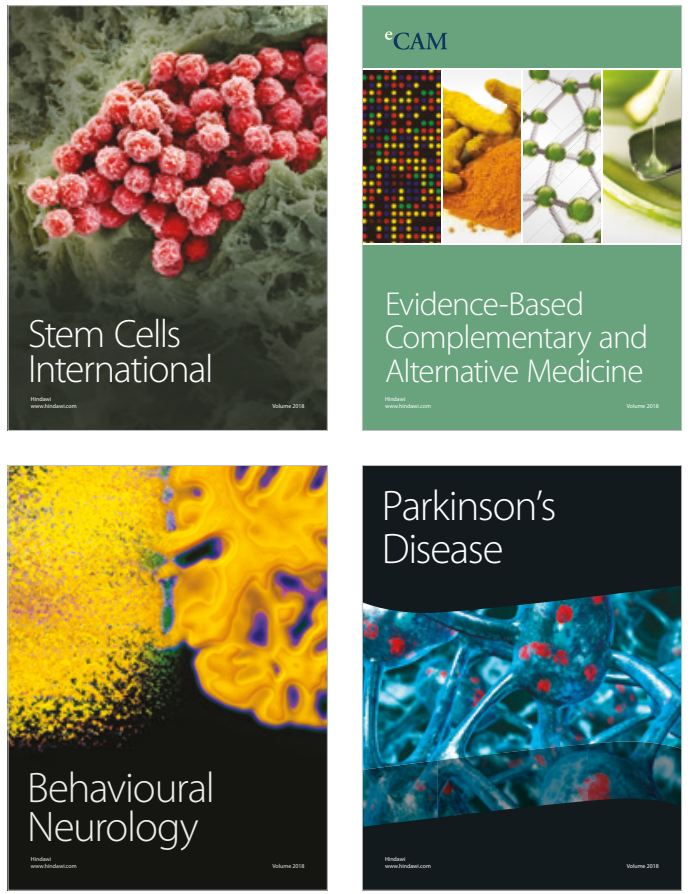

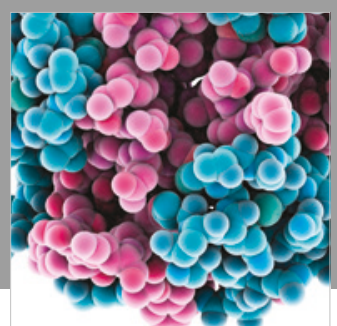

ournal of

Diabetes Research

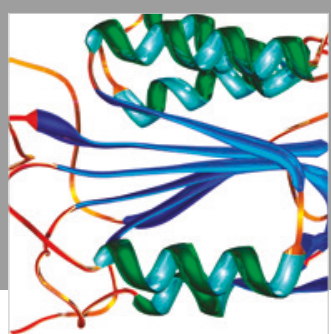

Disease Markers
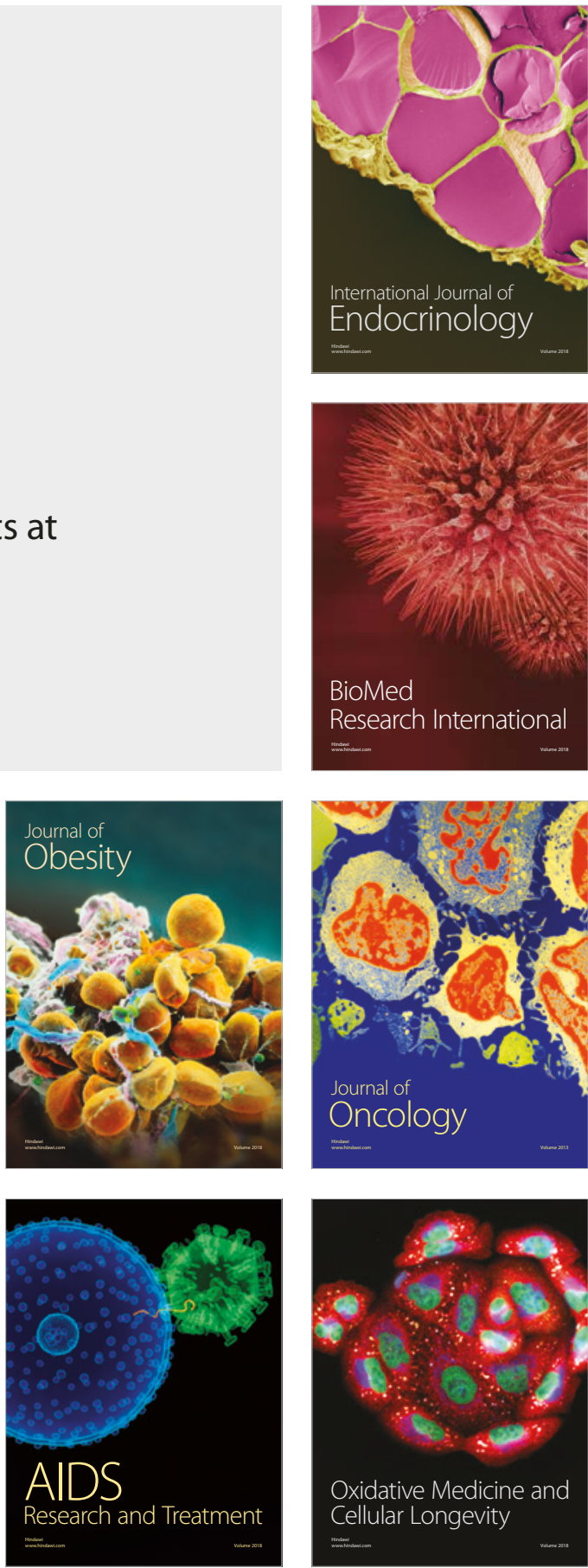